In: Raithel, J. (Hg): Risikoverhaltens im Jugendalter. Leske und Budrich, Opladen, 2001

Maria Limbourg, Jürgen Raithel, Karl Reiter

\title{
Jugendliche im Straßenverkehr
}

\section{Einleitung}

Die Entwicklung junger Menschen ist verbunden mit einer zunehmenden Ausdehnung ihrer Aktionsräume. Zunächst zu Fuß, später mit dem Fahrrad, mit dem motorisierten Zweirad und dann mit dem Auto gelingt es ihnen, ein immer größeres Umfeld zunehmend selbständiger zu erfahren. Hinzu kommen die Erfahrungen, die sie als Mitfahrer im Pkw und in öffentlichen Verkehrsmitteln bereits vom frühesten Kindesalter an gemacht haben. Der Erwerb der Pkw-Fahrerlaubnis und damit der Übergang vom Mitfahrer zum Fahrer ist für junge Menschen heute zu einer bedeutenden Übergangssituation vom Jugendlichen zum Erwachsenen geworden (vgl. Klein 2000).

Diese - aus psychologischer Sicht durchaus positive - Entwicklung im Jugendalter geht in der heutigen motorisierten Gesellschaft leider mit einem deutlich erhöhten Unfallrisiko einher (vgl. Tabellen 1 und 2). Die hohe Risikobereitschaft, die Selbstüberschätzung, die Vorstellung von "Freiheit und Abenteuer", Imponiergehabe und fehlende Erfahrung bilden ein vielfach fatales Bedingungsgefüge für den Verkehrsunfall im Jugendalter in allen hochindustrialisierten und deshalb auch hochmotorisierten - Ländern (vgl. Brühning et al. 1996).

Im Jugendalter vermischt sich das "jugendspezifische Risikoverhalten" mit dem "Anfängerrisiko" bei der Teilnahme am Straßenverkehr. Die Anfängerrisiken treten bei jenen auf, die erst seit kurzer Zeit gelernt haben, ein Fahrrad, ein Motorzweirad oder ein Auto zu fahren. In dieser Zeit neigen die Fahranfänger dazu, ihre Fertigkeiten zu überschätzen und die Gefahren des Straßenverkehrs zu unterschätzen (VCÖ 1999; Klein 2000). Darüber hinaus ist insbesondere das alterstypische Risikoverhalten als eine Hauptgefährdungsgröße zu sehen (vgl. Limbourg et al. 2000; Raithel 1999).

Jugendliche nutzen den Verkehrsraum außer zur funktionalen Fortbewegung als Sport- und Kommunikationsraum und Treffpunkt für Gruppenaktivitäten. In 
der Peer-Group - der wichtigsten sozialen Bezugsgruppe - werden jugendtypische riskante Verhaltensweisen gesteuert und übernehmen eine wichtige soziale Funktion (vgl. Jessor in diesem Band), über die der Jugendliche Anerkennung findet. Je nach Zweck und Art der Verkehrsteilnahme ergeben sich dabei unterschiedliche Gefährdungspotentiale, die im folgenden Abschnitt dargestellt werden.

\section{Verkehrsunfälle im Jugendalter}

\subsection{Verkehrsunfallstatistische Daten}

Verkehrsunfälle stellen die häufigste Todesursache im Jugendalter dar (Statistisches Bundesamt 1999a). Allein im Jahr 1998 starben in Deutschland insgesamt 1299 Jugendliche und junge Erwachsene im Alter von 15 bis unter 21 Jahren im Straßenverkehr. Weitere 22360 Personen dieser Altersgruppe wurden schwer und 69346 leicht verletzt (Statistisches Bundesamt 1999b).

Bei tödlichen Straßenverkehrsunfällen von Jugendlichen hat der Pkw als Verkehrsmittel eine herausragende Bedeutung: Die weitaus meisten Straßenverkehrstoten bei Jugendlichen sind Pkw-Insassen (siehe Tab. 1). In der Altersgruppe der 15- bis 17-jährigen sind Jugendliche in der Regel als Mitfahrer bzw. Mitfahrerinnen im Pkw unterwegs; in der Gruppe der 18- bis 21-jährigen fahren viele Jugendliche schon selbst einen Pkw. Betrachtet man nicht nur die absoluten Unfallzahlen, sondern auch die Verkehrsleistung (Verunglückte pro 1 Mio. gefahrene Kilometer), zeigt sich, dass das Pkw-Unfallrisiko mit 2 Verunglückten pro 1 Mio. Pkw-Kilometer in der Gruppe der 18-jährigen am größten ist. In Vergleich dazu beträgt das Unfallrisiko bei den 25- bis 44-jährigen Autofahrern nur 0,3 Verunglückte je 1 Mio. Kilometer (vgl. Limbourg et al. 2000, Kap. 3).

$\mathrm{Zu}$ den tödlichen Pkw-Unfällen kommen im Jugendalter noch die tödlichen Unfälle mit motorisierten Zweirädern (Statistisches Bundesamt 1999b). In dieser Verkehrsteilnehmergruppe liegt das verkehrsleistungsbezogene Unfallrisiko bei 40 Verunglückten pro 1 Mio. gefahrener Kilometer in der Gruppe der 15- bis 17jährigen Jugendlichen extrem hoch. Im Vergleich dazu liegt das Risiko in der Gruppe der 25- bis 44jährigen motorisierten Zweiradfahrer nur bei 3 Verunglückten je 1 Mio. Kilometer (vgl. Limbourg et al. 2000, Kap. 3). 
Das Risiko von Jugendlichen und jungen Erwachsenen auf den Schul- und Ausbildungswegen zeigen die Statistiken der Schülerunfallversicherungen auf (vgl. BUK 1997). Diese Unfallstatistiken weisen eine geringere Dunkelziffer als die Statistiken des Statistischen Bundesamtes auf (vgl. Limbourg et al. , 2000, Kap. 3). Aus diesem Grund geben die Schulwegunfall-Statistiken ein deutlicheres Bild über das Ausmaß der Fahrradunfälle im Jugendalter. Bei den amtlichen Polizeistatistiken über Fahrradunfälle beträgt die Dunkelziffer ca. 90\% (vgl. Hautzinger 1993).

In der Gruppe der 15- bis 17-jährigen Jugendlichen verunglückten im Jahr 199515771 Schülerinnen und Schüler auf dem Schulweg im Straßenverkehr, die meisten von ihnen (8815 Schüler/innen) mit dem Fahrrad, gefolgt von den Unfällen mit motorisierten Zweirädern (3492 Unfälle). Im Pkw verunglückten 941 Schülerinnen und Schüler, im Schulbus 670, im öffentlichen Verkehr 402 und zu Fuß 739. Insgesamt 17 Kinder dieser Altersgruppe wurden auf dem Schulweg getötet.

Bei den Schüler/innen im Alter ab 18 Jahren liegen die Unfälle mit dem Pkw (7781) an der Spitze, gefolgt von den Fahrradunfällen (4926). Mit "motorisierten" Zweirädern ereigneten sich 1782 Schulwegunfälle. Diese Altersgruppe verunglückt auf den Schulwegen auch am häufigsten tödlich (52 getötete Schüler/innen, 41 davon als Pkw-Insassen).

Tabelle 1: Anzahl der 1998 getöteten Jugendlichen und jungen Erwachsenen nach Alter, Geschlecht und Art der Verkehrsteilnahme (Stat. Bundesamt 1999b)

\begin{tabular}{|c|c|cc|cc|ccc|cc|cc|}
\hline Alter & gesamt & \multicolumn{2}{|c|}{ Mofa/Moped } & \multicolumn{2}{|c|}{ Motorräder } & \multicolumn{2}{|c|}{ PKW } & \multicolumn{2}{|c|}{ Fahrrad } & \multicolumn{2}{|c|}{ Fußgänger } \\
\hline 15 & 61 & 7 & 0 & 3 & 2 & 9 & 18 & 5 & 3 & 3 & 5 \\
\hline 16 & 132 & 14 & 3 & 34 & 5 & 26 & 34 & 4 & 2 & 4 & 6 \\
\hline 17 & 166 & 8 & 2 & 32 & 7 & 32 & 71 & 4 & 1 & 4 & 5 \\
\hline 18 & 364 & 4 & 1 & 22 & 1 & 250 & 67 & 6 & 0 & 9 & 2 \\
\hline 19 & 278 & 2 & 0 & 15 & 0 & 190 & 54 & 3 & 2 & 10 & 2 \\
\hline 20 & 298 & 0 & 0 & 30 & 4 & 180 & 67 & 2 & 1 & 10 & 1 \\
\hline
\end{tabular}

Wird das bevölkerungsbezogene Risiko von Jugendlichen nach einzelnen Altersstufen differenziert betrachtet, so wird deutlich, dass die 18- und 19-jährigen die höchsten Getötetenzahlen aufweisen. Differenziert man auch noch nach dem 
Geschlecht der Getöteten, zeigt sich eine extrem hohe Unfallbelastung bei den männlichen Jugendlichen.

Auch wenn man die Gesamtzahl der Verunglückten in dieser Altersgruppe betrachtet, sind die jungen Männer wesentlich stärker vertreten als die jungen Frauen (siehe Tab. 2). Eine Ausnahme bilden die Mädchen von 15 bis 17 Jahren: Sie verunglücken als Mitfahrerinnen im Pkw häufiger als gleichaltrige männliche Jugendliche - oft als Begleiterinnen von älteren jungen Männern, die schon einen Pkw-Führerschein besitzen.

Der große Unterschied zwischen Jungen und Mädchen zeigt sich auch bei der Betrachtung der Statistiken 1998 über das Geschlecht des Fahrzeugführers bei Unfällen mit Personenschäden (Getötete und Verletzte): In der Gruppe der 15- bis 18-jährigen sind es 12800 Jungen und nur 2489 Mädchen. In der Gruppe der 18- bis 21-jährigen ist das Verhältnis 46096 junge Männer zu 21089 junge Frauen (Statistisches Bundesamt 1999b). Ähnlich große Unterschiede zwischen Frauen und Männern zeigen sich auch bei der Betrachtung der Hauptverursacher von Verkehrsunfällen mit Verletzten und Getöteten in der Gruppe der 15- bis 20jährigen Jugendlichen und jungen Erwachsenen: Männer verursachen $53 \%$ der Mofa-, 49\% der Motorrad-, 52\% der Pkw-, 49\% der Fahrrad- und 43\% der Fußgängerunfälle selbst. Bei Frauen sind es nur 40\% der Mofa-, 33\% der Motorrad-, 49\% der Pkw-, 37\% der Fahrrad- und 31\% der Fußgängerunfälle (ebenda).

Tabelle 2: Anzahl der 1998 verunglückten (verletzen und getöteten) Jugendlichen und jungen Erwachsenen nach Alter, Geschlecht und Art der Verkehrsteilnahme (Statistisches Bundesamt 1999b)

\begin{tabular}{|c|c|cc|cc|cc|cc|}
\hline Alter & gesamt & \multicolumn{2}{|c|}{ Mofa/Moped } & \multicolumn{2}{|c|}{ Motorräder } & \multicolumn{2}{c|}{ PKW } & \multicolumn{2}{c|}{ Fahrrad } \\
& & $\mathrm{m}$ & $\mathrm{w}$ & $\mathrm{m}$ & $\mathrm{w}$ & $\mathrm{m}$ & $\mathrm{w}$ & $\mathrm{m}$ & $\mathrm{w}$ \\
\hline 15 & 6831 & 939 & 237 & 247 & 148 & 813 & 1340 & 1541 & 792 \\
\hline 16 & 13201 & 2301 & 674 & 3289 & 716 & 1330 & 2015 & 1488 & 712 \\
\hline 17 & 14868 & 2161 & 616 & 3360 & 714 & 2522 & 2843 & 1249 & 633 \\
\hline 18 & 21177 & 752 & 223 & 1324 & 341 & 9286 & 7034 & 1126 & 514 \\
\hline 19 & 19383 & 371 & 163 & 882 & 238 & 9049 & 6801 & 770 & 392 \\
\hline 20 & 17563 & 249 & 118 & 838 & 185 & 7961 & 6277 & 565 & 440 \\
\hline
\end{tabular}

In der Tabelle 2 ist auch zu erkennen, dass die Jugendlichen jeweils mit dem Verkehrsmittel sehr häufig verunglücken, das sie zum ersten Mal nutzen dürfen. 
Aus diesem Grund kann angenommen werden, dass neben der jugendspezifischen Risikobereitschaft auch das "Anfängerrisiko" eine wichtige Rolle bei der Verursachung von Verkehrsunfällen in dieser Altersgruppe spielt (vgl. VCÖ 1999; Limbourg et al. 2000).

Die häufigsten - bundesstatistisch erfassten - Fehlverhaltensweisen von Fahrern, die zu Unfällen (mit Personenschaden) führten, sind in Tabelle 3 dargestellt. Eine „nicht angepasste Geschwindigkeit“ ist die dominierende Unfallursache unter den motorisierten jungen Fahrenden, unter den Radfahrenden ist hingegen die falsche Straßenbenutzung die häufigste Fehlverhaltensweise.

Tabelle 3: Verteilung der Unfälle mit Personenschaden nach Fehlverhalten der 15- bis unter 18-jährigen Fahrrad- und Motorzweiradfahrer und der 18- bis unter 21-jährigen Autofahrer für das Jahr 1998 (vgl. Stat. Bundesamt 1999b)

\begin{tabular}{|c|c|c|c|}
\hline & $\begin{array}{l}\text { Fahrradfahrer } \\
\text { absolut (Rang) }\end{array}$ & $\begin{array}{l}\text { Motorzweirad- } \\
\text { fahrer } \\
\text { absolut (Rang) }\end{array}$ & $\begin{array}{c}\text { Autofahrer } \\
\text { absolut (Rang) }\end{array}$ \\
\hline $\begin{array}{l}\text { Nicht angepasste } \\
\text { Geschwindigkeit }\end{array}$ & 312 (4.) & 2259 (1.) & 16836 \\
\hline Vorfahrt/Vorrang & $655 \quad$ (3.) & 1109 & $6448 \quad(2)$. \\
\hline Abstand & - & - & $6196 \quad$ (3.) \\
\hline Abbiegen, Wenden & $681 \quad(2)$. & $948 \quad(3)$. & 5652 \\
\hline Überholen & $92 \quad(6)$. & $679 \quad(5)$. & 2190 \\
\hline $\begin{array}{c}\text { Falsche } \\
\text { Straßenbenutzung }\end{array}$ & 1414 (1.) & $886 \quad$ (4.) & 2874 \\
\hline Alkoholeinfluss & $104 \quad(5)$. & $318 \quad(6)$. & $2575 \quad(6)$. \\
\hline Sonstige & 1380 & 3331 & 10741 \\
\hline Insgesamt & 4638 & 9530 & 53512 \\
\hline
\end{tabular}

Weiterhin verunfallen die Fahrradfahrer aufgrund falschen Abbiegens und Wendens sowie Nichtbeachtens von Vorfahrt bzw. Vorrang. Bei den Motorzweiradfahrern (Mofa, Moped und Leichtkraftrad) ist das Nichtbeachten von Vorfahrt/Vorrang eines anderen Verkehrsteilnehmers, unkorrekte Abbiege- und Wendevorgänge sowie die falsche Straßenbenutzung häufige Unfallursachen. Die jungen Autofahrer verunglücken neben den dominanten „Geschwindigkeits- 
unfällen“ am meisten durch Vorfahrt- und Vorrangunfälle, zu geringem Abstand und bei Abbiege- und Wendevorgängen. Jeder fünfte Autofahrer zwischen 18 und 21 Jahren verunglückt aufgrund einer erhöhten Blutalkoholkonzentration.

Eine „neue“ Unfallgefahr für Jugendliche im Straßenverkehr stellt das InlineSkating dar. Diese Art der Fortbewegung wird in Deutschland immer beliebter. Laut Statistik fährt in Deutschland jeder siebte Inline-Skates (vgl. PFI 1998). Viele Skater nehmen dabei auch am Straßenverkehr teil (,street-skating“). Ungefähr $77 \%$ der Inline-Skater in Deutschland sind unter 20 Jahre alt. Sie erreichen häufig Geschwindigkeiten von 20-30 km/h mit Spitzen bis zu $50 \mathrm{~km} / \mathrm{h}$, haben jedoch ein eingeschränktes Bremsvermögen. Der Fahrstil der jugendlichen Inline-Skater reicht, je nach Fahrkönnen und Ambition, vom „Freizeit-Skating“ bis hin zum „Aggressive- und Stunt-Skating“ mit artistischen Sprüngen und Hindernisfahrten - häufig auf Treppen, Mauern und Bordsteinkanten. Da InlineSkating-Unfälle in den amtlichen Statistiken den Fußgängerunfällen zugeordnet werden, kann ihre Zahl nicht auf diesem Wege ermittelt werden. Es liegen allerdings inzwischen einige Studien aus Unfallkliniken vor, die über Inline-SkatingUnfälle Aufschluss geben. In diesen Studien sind allerdings nicht nur die Straßenverkehrsunfälle mit Inline-Skates zu finden, sondern auch Skating-Unfälle, die sich außerhalb des Straßenverkehrs ereignet haben.

In einer Studie am Universitätsklinikum Essen wurden im Jahr 1996 insgesamt 70 Inline-Skating-Unfälle untersucht (Majetschak et al. 1997). Das Alter der Verletzten lag zwischen 9 und 26 Jahren, mit einem Durchschnitt von 18 Jahren. $65 \%$ der verunglückten Skater waren männlich, 35\% weiblich. Die häufigsten Unfallursachen waren kleine Hindernisse (33\%) auf trockener und ebener Fahrbahn. Den höchsten Anteil schwerer Verletzungen wiesen Anfänger auf. In einer Studie von 1995 - 1996 wurden 196 Inline-Skating Unfälle von Kindern und Jugendlichen im Alter von 8 - 15 Jahren in Österreich erfasst (vgl. Schimpl 1997). Als Ursache für den Unfall wurden in $61 \%$ der Fälle Unebenheiten, in $16 \%$ Stürze beim Bremsen, in $85 \%$ Ausweichmanöver, in $6 \%$ eine zu hohe Geschwindigkeit und in $8 \%$ der Fälle Stürze beim Losfahren und Kollisionen angegeben. Die 196 Patienten erlitten 233 Verletzungen: 55\% hatten eine Fraktur; an zweiter Stelle folgte das Schädel-Hirn-Trauma mit 14\%. Aus diesem Grund ist das Tragen eines Schutzhelms bei dieser Sport- und Verkehrsart von großer Bedeutung für das Verletzungsrisiko der Jugendlichen. In einer Untersuchung aus Nordrhein-Westfalen (vgl. PFI 1998) wurden 152 Skating-Unfälle im Bereich 
des Straßenverkehrs analysiert (149 Inline-Skating-Unfälle und 3 SkateboardUnfälle). 75 Verletzte waren unter 15 Jahren, 77 waren 15 Jahre und älter. Bei 47 Unfällen wurden die Skater schwer verletzt. Männliche Skater verunglückten wesentlich häufiger als weibliche $(2: 1)$. Bei 88 Unfällen kollidierten die Skater mit einem Pkw, gefolgt von 32 Zusammenstößen zwischen Skatern und Radfahrern. Verursacher der Unfälle waren in 87 Fällen die Skater selbst, 35-mal die Pkw-Fahrer und 18-mal die Radfahrer.

Bei den Skating-Unfällen muss - wie auch bei den Fahrradunfällen - eine hohe Dunkelziffer angenommen werden, weil sich beim Skaten viele Alleinunfälle ereignen (Stürze). Eine empirische Untersuchung dazu liegt leider noch nicht vor.

\subsection{Einflussgrößen auf Verkehrsunfälle im Jugendalter}

Versucht man den typischen tödlichen Autounfall bei Jugendlichen und jungen Erwachsenen zu beschreiben, handelt es sich dabei um einen schweren Alleinunfall durch Kontrollverlust, der sich auf einer Freizeitfahrt mit Freunden unter Alkoholeinfluss bei hoher Geschwindigkeit am Wochenende in der Nacht ereignet (vgl. Keskinen 1996, Schulze 1998). Unter diesen Freizeitunfällen kommt den sog. "Disco-Unfällen" eine herausragende Bedeutung zu, welche die folgenschwersten nächtlichen Freizeitunfälle in dieser Altersgruppe darstellen.

Betrachtet man die Statistiken 1998 über alkoholisierte Verunglückte im Straßenverkehr, zeigt sich ein sehr großer Unterschied zwischen jungen Männern und jungen Frauen: In der Gruppe der 15- bis 18-jährigen kommen auf 660 männliche alkoholisierte Verunglückte nur 58 weibliche. Bei den 18- bis 21jährigen sind es 2895 Männer und nur 220 Frauen (vgl. Statistisches Bundesamt 1999b). Ein weiteres Problem in Zusammenhang mit der Verkehrsunfallproblematik im Jugendalter stellt der Konsum von Drogen dar (Polizeiblatt 2000). Mindestens jede 10. untersuchte Blutprobe enthält Drogen (Cannabis, Heroin, Kokain, Amphetamin (einschließlich Ecstasy, LSD) - oft zusammen mit Alkohol (vgl. Schulz et al. 1998).

Ein bedeutsamer Risikofaktor im Jugendalter ist die Selbstüberschätzung: 86\% der jungen Fahranfänger schätzen ihren Fahrstil als ,sicher“ ein (vgl. Shell/ADAC 2000). 
Weitere Risikofaktoren für das Zustandekommen von Verkehrsunfällen in dieser Altersgruppe sind der Lebensstil, die Persönlichkeit, der familiäre Hintergrund sowie die Schul- und Ausbildungssituation der Jugendlichen (vgl. Schlag et al. 1986; Keskinen 1996; Schulze 1996; 1999; Raithel 1999). Nach Lebensstilanalysen ist das Unfallrisiko für die sog. "Action-Typen", "Fan-Typen" und „Kicksuchende-Typen“ unter den Jugendlichen deutlich erhöht. Diese FreizeitTypen besuchen häufiger als andere Diskotheken oder Fußballspiele und trinken dabei auch viel Alkohol. Deshalb verunglücken sie häufig im Straßenverkehr. Ungefähr $37 \%$ der 18 - bis 24jährigen - vorwiegend männlichen - jungen Erwachsenen können diesen Gruppen zugeordnet werden (vgl. Schulze 1996; 1999).

Verkehrsunfälle im Jugendalter - und besonders nächtliche Disco-Unfälle gehen mit einer niedrigen schulischen Qualifikation einher: Circa 65\% der Verursacher von Disco-Unfällen sind Hauptschüler, obwohl ihr Anteil in der Altersgruppe nur $37 \%$ beträgt. Sie sind demnach unter den Verursachern nächtlicher Freizeitunfälle deutlich überrepräsentiert. In den Berufsschulen sind Lehrlinge aus den Berufen Metall und Bau stärker unfallgefährdet als andere Berufsgruppen. Fast jeder zweite nächtliche Freizeitunfall (48\%) wird von Angehörigen dieser beiden Berufsgruppen verursacht (vgl. Marthiens/Schulze 1989, Schulze 1998).

Zu der Risikogruppe “Junge Fahrer" zählen besonders solche Jugendliche, die auf psychologischer Ebene weniger durch eine rationale als durch eine emotionale Verhaltenssteuerung und ein zu großes subjektives Sicherheitsgefühl (Selbstüberschätzung) gekennzeichnet sind (vgl. Schulze 1998). Auch "hyperaktive" Jugendliche sind stärker unfallgefährdet (vgl. Barkley et al. 1993).

Besonderen Risiken im Straßenverkehr sind auch sog. "Problem-Kids" ausgesetzt (vgl. Henning et al. 1996). Sie haben häufig einen Freundeskreis, in dem motorisiertes Zweiradfahren "in" ist und oft Alkohol getrunken und riskant gefahren wird. Als Konsequenz fährt der Jugendliche selber alkoholisiert und riskant - später auch mit dem Auto. Eine hohe Anzahl von erlebten Unfällen in der gesamten Verkehrsgeschichte ist die Folge.

Betrachtet man die Gruppe der jugendlichen Motorzweiradfahrer, so ist erst einmal eine "dispositionell” höhere Risikobereitschaft im Vergleich zu den nichtmotorzweiradfahrenden Altersgenossen festzustellen (vgl. Raithel 1999). Besonders verkehrsriskant verhalten sich dann diejenigen Motorzweiradfahrer, 
die fest in einer Peer-Group integriert sind, eine hohe Selbstwirksamkeitserwartung aber geringe Selbstwertschätzung haben, die durch schulische Anforderungen stark gefordert sind, denen es an familiärer Unterstützung mangelt und die in einem konfliktreichen Elternhaus leben. Psychosoziale Belastungen während der Statuspassage (vgl. Schnabel in diesem Band) wirken dementsprechend als eine Einflussgröße auf das riskante Verkehrsverhalten (vgl. Schuman et al. 1967, Brown et al. 1986; Jessor 1987; Raithel 1999). Als verkehrssicherheitsabträglich hat sich hingegen das Motorzweiradfahren in der Gruppe nicht herausgestellt, somit kann mit dem weitläufigen Vorurteil, dass das Motorzweiradfahren in Gruppen den Leichtsinn und die Riskanz des Fahrstils verstärken, aufgeräumt werden. Denn im Gegenteil fahren nur solche Jugendlichen in der Gruppe, deren Fahrstil rücksichtsvoll und angepasst ist. Ein riskant fahrender Jugendlicher würde überhaupt nicht in einer Gruppe mitfahren dürfen. Die Gruppe bringt auch einen passiven Verkehrssicherheitsgewinn, da diese von anderen Verkehrsteilnehmern viel besser wahrgenommen werden kann als ein einzelner Fahrer (vgl. Raithel 1999).

Ein Vergleich zwischen Fahrrad-, Mofa- und Leichtkraftradfahrern zeigte, dass die jugendlichen Fahrradfahrer öfter gegen Verkehrsvorschriften verstoßen und sich häufiger offensiv-riskant im Verkehr verhalten als ihre motorzweiradfahrenden Altersgenossen. Hingegen verhalten sich die jugendlichen Motorzweiradfahrer verantwortungsvoller und verkehrsregelangepasster als die radfahrenden Jugendlichen (vgl. Raithel 2000). Trotzdem verunfallen straßenverkehrsstatistisch gesehen häufiger Mofafahrer als Fahrradfahrer (vgl. Tab. 2) und männliche Fahrer öfter als weibliche. Für diesen Unterschied geben Hubacher und Ewert (1994) sicherheitsabträgliche Einstellungen (hohe Risikobereitschaft und häufiges Missachten von Verkehrsregeln) von zweiradfahrenden Jungen und die mangelhafte Fahrzeugbeherrschung von Zweiradfahrerinnen an. Das Verkehrsverhalten von jugendlichen Mofafahrern hängt besonders von den ,personenbezogenen“ Faktoren Verkehrsregelwissen, Gefahrenkognition, Einstellungen und emotionaler Verfassung ab, wobei eine schlechte Fahrzeugbeherrschung (Anfängerrisiko) nicht die hohe Unfallbelastung zu verantworten hat (vgl. Koch 1980).

Als Hauptunfallursachen Jugendlicher im Straßenverkehr lassen sich zusammenfassend die hohe jugendspezifische Risikobereitschaft, nicht angepasste Geschwindigkeit, Alkohol- und Drogengebrauch, Imponierverhalten, familiäre und 
schulische Schwierigkeiten sowie mangelnde Fahrerfahrung und Delinquenz (Brown et al. 1986) benennen. Die Mitfahrerbeeinflussung stellt weiterhin ein Risiko dar, welches sich jedoch für die Motorzweiradfahrer weitgehend ausschließt (vgl. Raithel 1999). Hingegen ist ,,aufputschende“ und laute Musik (vgl. Hanel in diesem Band) im Auto als ein Verkehrsrisiko zu sehen.

\section{Delinquentes Verhalten von Jugendlichen im Straßenverkehr}

Delinquentes Verhalten von Verkehrsteilnehmer/innen wird beim KraftfahrtBundesamt (KBA) im Verkehrszentralregister (VZR) registriert und kann neben dem Unfallgeschehen als ein weiterer Indikator für Risikobereitschaft im Straßenverkehr herangezogen werden. Die VZR-Statistik umfasst Daten zu Delikten wie z.B. Fahren ohne Fahrerlaubnis, Alkoholgenuss beim Führen des Fahrzeugs, Verstöße bezüglich Geschwindigkeit, Vorfahrt, Überholen, Sicherheitsabstand, Abbiegen usw. Im VZR sind Jugendliche ab 14 Jahren vertreten, weil nach dem Strafgesetzbuch mit diesem Alter die Strafmündigkeit beginnt (vgl. Hansjosten 1999).

Betrachtet man die Anzahl der im VZR registrierten jugendlichen Täter je 1000 Halter von Kraftfahrzeugen (Krafträder und Pkw) im VZR zeigt sich, dass die Jugendlichen eine Spitzenposition einnehmen. Männliche Jugendliche sind dabei sehr viel stärker vertreten als weibliche (siehe Tab. 4).

Tabelle 4: Täter im VZR je 1.000 Fahrzeughalter und je 100.000 Personen der Altersgruppe nach Alter und Geschlecht im Jahr 1997 (vgl. Hansjosten 1999)

\begin{tabular}{|c|rr|rr|cc|}
\hline Alter & \multicolumn{2}{|c|}{ Halter von KFZ } & \multicolumn{2}{|l|}{ Täter je 1000 Halter } & \multicolumn{2}{|c|}{$\begin{array}{c}\text { Täter je 100 000 Perso- } \\
\text { nen der Altersgruppe }\end{array}$} \\
\hline & männlich & weiblich & männlich & weiblich & männlich & weiblich \\
$14-17$ & 27479 & 15747 & 619 & 64 & & 798 \\
$18-20$ & 349862 & 165251 & 389 & 182 & 12013 & 27343 \\
$21-24$ & 1094682 & 559568 & 217 & 109 & 24214 & 6340 \\
\hline
\end{tabular}


Mit zunehmendem Alter verringert sich die Anzahl der Täter je 1000 Fahrzeughalter deutlich und auch der Unterschied zwischen Männern und Frauen wird geringer. Bei diesen Zahlen muss allerdings berücksichtigt werden, dass mehr jüngere Personen und Frauen einen Pkw nutzen, den sie nicht besitzen.

Bei der Betrachtung der Gruppe der jungen Fahrer und Fahrerinnen zeigt sich auch noch, dass es jedem siebten Fahranfänger (14\%) nicht gelingt, die derzeit zweijährige Probezeit ohne eine Eintragung im VZR zu bestehen. Männer sind davon durchschnittlich 3,5mal häufiger als Frauen betroffen (vgl. Hansjosten/Schade 1997; Hansjosten 1999; Klein 2000). Dieses Verhältnis verschiebt sich noch weiter zu Lasten der Männer bei der Betrachtung von Verkehrsstraftaten, Alkohol und Geschwindigkeitsdelikten. $40 \%$ der während der Probezeit begangenen Delikte tragen einen Unfallvermerk, weit mehr als beim Durchschnitt der Kraftfahrer. Es bestätigt sich bei den Fahranfängern ein besonders enger Zusammenhang zwischen Verkehrsdisziplin und Verkehrssicherheit. 29\% der in der Probezeit auffällig gewordenen Fahranfänger der Klasse 3 werden innerhalb von zwei Jahren erneut in das VRZ eingetragen. Bei den Männern im Alter zwischen 19 und 24 Jahren beträgt die Rückfallquote sogar 45\% (vgl. Hansjosten/Schade 1997).

Delinquentes Verhalten im Bereich der Motorzweiräder ist vor allem im Auffrisieren und in der damit einhergehende Überschreitung der fahrzeugklassenspezifischen Höchstgeschwindigkeit zu finden. Besonders auffällig sind hierbei die Mofafahrer: 52 Prozent der Mofafahrer haben ihr Zweirad auffrisiert und jeder befragte Jugendliche fuhr mit seinem Mofa schneller als die zulässigen $25 \mathrm{~km} / \mathrm{h}$. Immer noch 40 Prozent der Mofafahrer fahren mit ihrem Motorzweirad Höchstgeschwindigkeiten von über $50 \mathrm{~km} / \mathrm{h}$. Die „Auffrisier-Quote“ liegt bei den Mopeds und Leichtkrafträdern um 38 Prozent. Unter den Fahrern von Mopeds, Mokicks und Roller bis $50 \mathrm{ccm}$ gibt es immerhin schon rund 10 Prozent, deren Fahrzeug die vorgeschriebene Höchstgeschwindigkeit von $50 \mathrm{~km} / \mathrm{h}$ nicht überschreitet. Dennoch kommen ein Viertel der Mopedbesitzer zu Höchstgeschwindigkeiten von mind. $70 \mathrm{~km} / \mathrm{h}$. Leichtkraftradbesitzer dürfen bis zu $80 \mathrm{~km} / \mathrm{h}$ fahren. Mit dieser vorgeschriebenen Höchstgeschwindigkeit geben sich auch 17 Prozent der Jugendlichen zufrieden, aber immer noch ein gutes Drittel fährt mit über 110 $\mathrm{km} / \mathrm{h}$ (vgl. Raithel 1999). 


\section{Prävention}

Zur Verringerung der Unfallrisiken für Jugendliche und junge Erwachsene können unterschiedliche Maßnahmen getroffen werden (vgl. Limbourg et al. 2000, Kap. 6). Die in Frage kommenden Maßnahmen lassen sich folgenden Kategorien zuordnen:

- $\quad$ planerische und technische Maßnahmen und Normierung (engineering),

- $\quad$ legislative Maßnahmen, Kontrolle und Überwachung (enforcement),

- Anreizsysteme (economy)

- $\quad$ kommunikative und erzieherische Maßnahmen (education).

Planerische und technische Maßnahmen haben häufig eine hohe und dauerhafte Wirkung auf die Erhöhung der Verkehrssicherheit. So sind z. B. verkehrsverlangsamende bauliche Maßnahmen (Geschwindigkeitsbeschränkungen, Fahrbahneinengungen, Aufpflasterungen, Kreisverkehre usw.) wirksame „Dauerbremsen“ für den Straßenverkehr - und auch für junge Fahrer. Auch die Einrichtung von Discobus-Linien und der Ausbau des nächtlichen öffentlichen Verkehrs können zur Erhöhung der Verkehrssicherheit von jungen Fahrern beitragen, wenn diese vom Auto auf den Bus umsteigen. Ähnliches gilt für den Ausbau der Radwegnetze in den Städten und Gemeinden.

Auch die Gesetzgebung kann einen wichtigen Beitrag zur Verbesserung der Verkehrssicherheit von jungen Fahrern leisten. So war z.B. die Einführung der Helmpflicht für motorisierte Zweiradfahrer ein deutlicher Beitrag zur Erhöhung der Sicherheit von Motorzweiradfahrern. Ähnliches gilt für die Gurtpflicht im Pkw oder für die Herabsetzung der Promillegrenze für Alkohol von 0,8 auf der 0,5. Auch die Einführung des Führerscheins auf Probe war ein - leider nicht besonders erfolgreicher - Versuch, die Verkehrssicherheit dieser Altersgruppe zu erhöhen (vgl. Klein 2000).

Verkehrsregeln können allerdings nur dann wirksam werden, wenn sie auch eingehalten werden. Aus diesem Grund sind Kontrolle und Überwachung zur Erhöhung der Verkehrssicherheit von großer Bedeutung. Insbesondere sind Verkehrsregeln, die eine Auswirkung auf die Sicherheit von Jugendlichen haben, durch eine gezielte Überwachung durchzusetzen (z.B. Alkohol-, Drogen- und Geschwindigkeits-Kontrollen in der Nähe von Discotheken, Kontrolle von „frisierten“ Mofas usw.). Und auch die sicherheitsorientierte nächtliche Überwa- 
chung in Bussen und Bahnen und an Haltestellen kann eine unfallpräventive Wirkung haben, weil dann mehr Jugendliche - und insbesondere mehr junge Frauen - den öffentlichen Verkehr, anstatt des Autos nutzen werden.

Unfälle können auch durch unterschiedliche Anreizsysteme verhindert werden. So könnten z. B. günstige Anfangstarife bei der Haftpflichtversicherung und eine starke Steigerung nach jedem Unfall zu einer vorsichtigeren Fahrweise motivieren. Die Nutzung von Disco-Bussen oder Sammeltaxen könnte durch ein Freigetränk ,,belohnt“" werden. Auch die Getränkepreise in Bars und Discotheken könnten sich verhaltenssteuernd auswirken (nicht-alkoholische Getränke sollten preiswerter als alkoholische Getränke sein).

Durch Planung, Technik, Regelung und Überwachung lassen sich viele, aber längst nicht alle Risiken für Jugendliche ausschalten. Aus diesem Grund sollen Kinder und Jugendliche in den verschiedenen Erziehungs- und Bildungsinstitutionen (Familie, Kindergarten, Grundschule, weiterführende Schulen) rechtzeitig lernen, Risiken richtig einzuschätzen und Gefahrensituationen zur vermeiden oder zu bewältigen. Zugleich sollen sie mit zunehmendem Alter ein kritisches Verständnis für den Verkehrsablauf und seine Komponenten erlangen und damit befähigt werden, an der Verbesserung der Verkehrsverhältnisse und an der Prävention von verkehrsbedingten Unfällen und Verletzungen mitzuwirken. Diese pädagogischen Erziehungs- und Bildungsansätze lassen sich als „Verkehrserziehung“, „Mobilitätserziehung“, „Gesundheitserziehung“ oder „Sicherheitserziehung“ bezeichnen (vgl. Limbourg et al. 2000, Kap. 6). „Verkehrs- und Mobilitätserziehung“ als „Sicherheitserziehung“ umfasst alle pädagogischen Maßnahmen, die den Menschen befähigen, mit den Gefahren in seiner Lebensumwelt umzugehen und sich für die Unfallverhütung einzusetzen.

Aufgabe der Mobilitäts- und Verkehrserziehung als Sicherheitserziehung ist es daher, Kindern und Jugendlichen alle jene Qualifikationen zu vermitteln, die sie für ein sicherheitsbewusstes Verhalten im Verkehrsraum benötigen. Dabei soll es nicht nur um den Erwerb von Kompetenzen zur Bewältigung der derzeitigen Gefahren im Verkehrsraum, sondern auch um das Erlernen von Strategien zur aktiven Veränderung der derzeitigen Situation, z. B. durch Beteiligung an den Stadt- und Verkehrsplanungsprozessen in den Städten und Gemeinden, gehen.

Im Rahmen dieser unfallpräventiven Sicherheitserziehung sollten alle für Kinder und Jugendliche relevanten Verkehrsarten (Fußgängerverkehr, Fahrradverkehr, Inline-Skating-Verkehr, Mofa-, Moped- und Motorradverkehr, Autover- 
kehr, öffentlicher Verkehr usw.) im Unterricht behandelt werden. Die Klassenstufenbezüge ergeben sich aus dem Mobilitätsverhalten und aus der Unfallbelastung der einzelnen Altersstufen mit verschiedenen Verkehrsunfalltypen. Dabei muss der entsprechende Verkehrs- und Mobilitätsunterricht immer ein bis zwei Jahre vor der Nutzung der einzelnen Verkehrsmittel angeboten und auch noch in der ersten Phase der neuen Art der Verkehrsteilnahme begleitend weitergeführt werden. Die Intensität des Verkehrstrainings muß dem Gefahrenpotential der Verkehrsmittelnutzung für die entsprechende Altersgruppe angepasst werden.

Die „Gesundheitserziehung“ umfasst alle pädagogischen Maßnahmen, die eine gesundheitsbewusste Lebensführung von Kindern und Jugendlichen zum Ziel haben. Dadurch sollen Krankheiten und Verletzungen verhindert und eine optimale physische, psychische und soziale Entwicklung in der Kindheit und Jugend ermöglicht werden. Das Ziel der Mobilitäts- und Verkehrserziehung als „Gesundheitserziehung“ ist es somit, den Schülerinnen und Schülern die verkehrsbedingten Risiken für die Gesundheit zu vermitteln und eine gesundheitsbewusste Verkehrsmittelwahl und -nutzung zu fördern.

Die Erziehungs- und Aufklärungsbemühungen im Rahmen der sicherheits- und gesundheitsorientierten Verkehrserziehung im Jugendalter sollten sich auf die Themen „Risikobereitschaft“ und „Risikoverhalten“ bei der Nutzung von Fahrrädern, Mofas, Mopeds, Motorrädern und Pkw konzentrieren. Besonders intensiv sollten diese pädagogischen Bemühungen bei der Arbeit mit den bekannten Risikogruppen liegen (Hauptschüler und Berufsschüler mit Ausbildungsberufen aus den Gebieten „Metall“" und „Bau“).

Möglichkeiten zur Kommunikation und Aufklärung bieten sich außer in der Schule und im Elternhaus auch besonders in Vereinen und Freizeiteinrichtungen, die von Jugendlichen häufig besucht werden (z.B. Fußballvereine, Discotheken, Popkonzerte). Die Sport-, Musik- und Film-Idole der Jugendlichen können dabei durch ihre Vorbildfunktion einen wichtigen Beitrag bei der Kommunikation von unfallpräventiven Botschaften leisten.

Auf der Grundlage entwicklungspsychologischer und sozialisationstheoretischer Erkenntnisse über das Jugendalter (vgl. Limbourg 1996; Raithel 1999) muss eine Perspektiverweiterung für die bisherige Verkehrserziehung, die vor allem über wenig effektive Wissensvermittlung und Abschreckung durch mediale Präsenta- 
tion von Unfallszenarien auf ein risikovermeidendes Verkehrsverhalten abzielen, gefordert werden.

Ein Verkehrssicherheitsbeitrag kann dadurch geleistet werden, dass an der Unfallursache, nämlich dem jugendlichen Risikoverhalten, angesetzt wird. Verkehrserziehung muss unter einer gesundheitspädagogischen Sicht betrieben werden (vgl. Limbourg et al. 2000, Kap. 6), um so das riskante Verkehrsverhalten im Kanon anderer jugendlicher Risikoverhaltensweisen zu reduzieren. Durch die Entwicklung, Stärkung und Etablierung geeigneter und "produktiver" Verhaltensweisen zur Problem- und Belastungsbewältigung eröffnet sich eine Chance zur Verringerung der Verkehrsgefährdung und Haupttodesursache Jugendlicher (vgl. Raithel 1999). Die Jugendlichen müssen lebensweltnahe erreicht werden, hier sind auch Gespräche mit gleichaltrigen verunglückten Rad-, Motorrad- oder Autofahrern in der Schulen oder in Unfall- oder Rehabilitationskliniken - als erfolgversprechend anzusehen (vgl. Vestergaard, 1996) Über Betroffenheit und Einsicht kann bei den Jugendlichen in aufdeckenden und nachhaltigen Maßnahmen eine Verhaltensänderung in präventiver Absicht erreicht werden.

\section{Literaturverzeichnis}

Barkley, R.A./Guevremont, D.C./Anastopoulus, A.D./Paul, G.J./Shelton, T.L. (1993): Driving related risks and outcomes of attention deficit hyperactivity disorder in adolescents and young adults: A three- to five-year follow-up survey. In: Pediatrics, 92, 212-218.

Brown, R.S./Sanders, J.M./Schonberg, S.K. (1986): Driving safety and adolescent behavior. In: Pediatrics, 77, 603-607.

Brühning, E./Kühnen, M.A./Berns, S. (1996). Verkehrssicherheit junger Leute international. In Bundesanstalt für Straßenwesen (Hg.): Junge Fahrer und Fahrerinnen. Bericht der Bundesanstalt für Straßenwesen, Heft M 52, Bergisch Gladbach, 72-81.

Bundesverband der Unfallversicherungsträger der öffentlichen Hand (2000): Straßenverkehrsunfälle in der Schüler-Unfallversicherung 1998. München.

Hansjosten, E. (1999): Delinquentes Verhalten im Straßenverkehr. In: Flade, A./Limbourg, M. (Hg.): Frauen und Männer in der mobilen Gesellschaft. Opladen: Leske + Budrich, 115-128.

Hansjosten, E./Schade, F.-D. (1997): Legalbewährung von Fahranfängern. Bergisch Gladbach: Bericht der Bundesanstalt für Straßenwesen, Heft M 71.

Hautzinger, H. (1993): Dunkelziffer bei Unfällen mit Personenschaden. Bergisch Gladbach: Bundesanstalt für Straßenwesen. 
Henning, H.-J./Lange, C./Chaselon, F. (1996). Verkehrsbiographische Entwicklungstypen junger Fahrer. In: Bundesanstalt für Straßenwesen (Hg.): Junge Fahrer und Fahrerinnen, Bergisch Gladbach: Bundesanstalt für Straßenwesen, Heft M 52, 104-117.

Hilsenbeck, Th. (1997): Die Alkoholfahrt junger Verkehrsteilnehmer: Risikofaktoren und informelle soziale Kontrolle. Göttingen: Cuvillier Verlag.

Hubacher, M./Ewert, U. (1994): Einstellungen und Merkmale der Fahrzeugbenutzung jugendlicher Velo- und Mofafahrer. Bern: Schweizerische Beratungsstelle für Unfallverhütung.

Jessor, R. (1987): Risky driving and adolescent problem behavior: An extension of problem-behavior theory. In: Alcohol, Drugs, and Driving, 3, 1-11.

Keskinen, E. (1996): Warum ist die Unfallrate junger Fahrerinnen und Fahrer höher? In: Bundesanstalt für Straßenwesen (Hg.): Junge Fahrer und Fahrerinnen. Bergisch Gladbach: Bundesanstalt für Straßenwesen, Heft M 52, 42-55.

Klein, P. (2000): Straßenverkehrsunfälle junger Menschen. Deutsches Polizeiblatt, 3, 4-8.

Koch, H. (1980): Verkehrswissen und -verhalten jugendlicher Mofafahrer. Bergisch Gladbach: Bundesanstalt für Straßenwesen.

Kraftfahrt-Bundesamt (1999): Verkehrsverstöße von Pkw-Fahrerinnen und -Fahrern. Flensburg.

Limbourg, M. (1996): Entwicklungspsychologische Grundlagen der Beeinflussung des Verhaltens von Jugendlichen. In: Bericht der Bundesanstalt für Straßenwesen (Hg.): Junge Fahrerinnen und Fahrer. Bergisch Gladbach, Heft M 52, 164-168.

Limbourg, M./Flade, A./Schönharting, J. (2000): Mobilität im Kindes- und Jugendalter. Opladen: Leske + Budrich.

Majetschak, M./Kock, H.-J./Neudeck, F./Schmit-Neuerburg, K.P. (1997): Unfallursachen und Verletzungsmuster bei Inline-Skating. In: Unfallchirurgie, 4, 171-178.

Marthiens, W./Schulze, H. (1989): Analyse nächtlicher Freizeitunfälle junger Fahrer: Discounfälle. In: Discounfälle - Fakten und Lösungsstrategien. Bergisch Gladbach: Bericht der Bundesanstalt für Straßenwesen, Köln, 1-74.

Polizeiblatt (2000): Junge Fahrer und Drogen - eine permanente Gefahr, 3, 25-28.

Polizei-Fortbildungs-Institut-Neuss (1998). Verkehrsunfälle mit Skatern in NordrheinWestfalen. Neuss.

Raithel, J. (1999): Unfallursache: Jugendliches Risikoverhalten. Verkehrsgefährdung Jugendlicher, psychosoziale Belastungen und Prävention. Weinheim/München: Juventa.

Raithel, J. (2000): Fahrmotive und Verkehrsverhalten jugendlicher Zweiradfahrer. Ein Vergleich zwischen männlichen Fahrrad-, Mofa- und Leichtkraftradfahrern. In: Zeitschrift für Verkehrserziehung 50, 2, im Druck.

Shell/ADAC (2000): Junge Fahranfänger. Hamburg \& München.

Schimpl, G. (1997). Inline-Skating Unfälle bei Kindern. In Kuratorium für Verkehrssicherheit: Bericht über die Internationale Konferenz "Mobilität \& Sicherheit" im Oktober. Wien. 
Schlag, B./Ellinghaus, D./Steinbrecher, J. (1986): Risikobereitschaft junger Fahrer. Bergisch Gladbach: Bundesanstalt für Straßenwesen, Heft 58.

Schulz, E./Vollrath, M./Klimesch, C./Szegedi, A. (1998): Fahruntüchtigkeit durch Cannabis, Amphetamine und Kokain. Bergisch Gladbach: Bericht der Bundesanstalt für Straßenwesen, Heft M 81.

Schulze, H. (1996): Lebensstil und Verkehrsverhalten junger Fahrer und Fahrerinnen. Bergisch Gladbach: Bundesanstalt für Straßenwesen, Heft M 56.

Schulze, H. (1998), Nächtliche Freizeitunfälle junger Fahrerinnen und Fahrer. Bergisch Gladbach: Bundesanstalt für Straßenwesen, Heft M 91.

Schulze, H. (1999): Lebensstil, Freizeitstil und Verkehrsverhalten 18- bis 34jähriger Verkehrsteilnehmer. Bergisch Gladbach: Bundesanstalt für Straßenwesen, Heft M 103.

Schuman, S.H./Pelz, D.C./Ehrlich, N.J./Selzer, M.L. (1967): Young male drivers. In: Jounal of American Medical Association, 200, 12, 1026-130.

Statistisches Bundesamt (1999a): Todesursachen 1998. Wiesbaden: Metzler Poeschel.

Statistisches Bundesamt (1999b): Straßenverkehrsunfälle 1998. Wiesbaden: Metzler Poeschel.

Verkehrsclub Österreich (VCÖ) (1999): Jugend und Verkehr. Wien.

Vestergaard, J. R. (1996): Verkehrsinformanten - Junge Leute mit einer Botschaft.In: Bericht der Bundesanstalt für Straßenwesen (Hg.): Junge Fahrerinnen und Fahrer. Bergisch Gladbach, Heft M 52, 224-227.. 